\title{
Searches for the direct pair production of third generation squarks using the ATLAS detector
}

\author{
John Anders ${ }^{1 \mathrm{a}}$ on behalf of the ATLAS Collaboration \\ ${ }^{1}$ The University of Liverpool
}

\begin{abstract}
Third generation squarks are expected to have masses of $<1 \mathrm{TeV}$ due to naturalness arguments. As such production of these squarks may be possible at the LHC. An overview of the most recent ATLAS third generation results and summary paper are presented in these proceedings. In addition to this, an overview of the outlook for such searches in Run 2 of the LHC is also presented.
\end{abstract}

\section{Introduction}

Supersymmetry (SUSY) is a possible extension of the Standard Model (SM) which provides solutions to questions which remain unanswered by the SM, such as the Higgs hierarchy problem, and certain SUSY scenarios also provide a candidate for dark matter [1].

The introduction of a scalar top quark $(\tilde{t})$ with mass $<1 \mathrm{TeV}$ allows for cancellations of the quantum loop corrections to the Higgs mass from the top quark, thus solving the hierarchy problem. Further to this, in R-parity conserving (RPC) SUSY models the lightest SUSY particle (LSP) is stable and a dark matter candidate. In most generic searches the LSP is a neutralino $\left(\tilde{\chi_{1}^{0}}\right)$ which is a superposition of the eigenstates corresponding to the Higgsinos and Bino. The main signature of such scenarios is a large amount of missing transverse momentum, $\mathrm{E}_{\mathrm{T}}^{\text {miss }}$ due to the non-interacting LSP leaving the detector. In R-parity violating scenarios (RPV) where the LSP is not stable and can decay to SM particles, this is not the case and vastly different signatures are expected involving a high object multiplicity.

\section{Overview of Recent Run 1 Analyses}

A wide variety of SUSY scenarios were searched for during Run 1 of the LHC. The general analysis strategy is as follows:

Signal regions (SRs) are defined targeting a specific model and SUSY mass parameter set. Selections based on key kinematic variables are optimised such that they maximise the discovery or exclusion significance for a particular model. Control regions (CRs) are then defined to constrain the main SM background processes present in the SR. These regions are kinematically close to the SR but are designed to be orthogonal to the SR, and are enriched in the specific background process under consideration. The number of events in the $\mathrm{CR}$ is used to produce normalisation parameters for the

a e-mail: john.kenneth.anders@cern.ch 
backgrounds in the SR. The normalisation parameters are validated in validation regions (VRs) which are also orthogonal to both the SRs and CRs. After validation the yields in the SRs are compared to the corrected SM prediction and if no excesses are observed, exclusion limits are placed on the models under consideration.

\subsection{Search for direct charm squark pair production}

A search for scharm quark pair production is presented in Ref. [2]. The analysis targets final states with two charm-tagged jets (tagged using a dedicated charm tagging algorithm with efficiency $O(20 \%))$ and large $\mathrm{E}_{\mathrm{T}}^{\text {miss }}$ to search for $\tilde{c} \rightarrow c+\tilde{\chi}_{1}^{0}$. As this analysis is a dedicated search for scharm quarks, the scharm quarks are treated as non-degenerate with respect to the other squarks. The main discriminating variable is the boost-corrected contransverse $\mathrm{m}_{\mathrm{CT}}$ [3], which is used to discriminate between scharm pair production and $t \bar{t}$ pair production.

Three overlapping SRs are defined, each with increasingly tighter $\mathrm{m}_{\mathrm{CT}}$ selections (the tighter the selection, the higher the $\tilde{c}$ mass targeted). The main SM processes that contribute to the SM background in the SRs are $t \bar{t}, Z$-jets and $W$-jets production. For each of these backgrounds a dedicated CR region is defined, which is enriched in the background under consideration.

No excesses are seen, and exclusion limits are placed in the $\left(\tilde{c}, \tilde{\chi_{1}^{0}}\right)$ mass plane, Fig. 1. The analysis extends the general exclusion for charm squarks originally set by the ATLAS 0L+2-6 jets analysis [4].

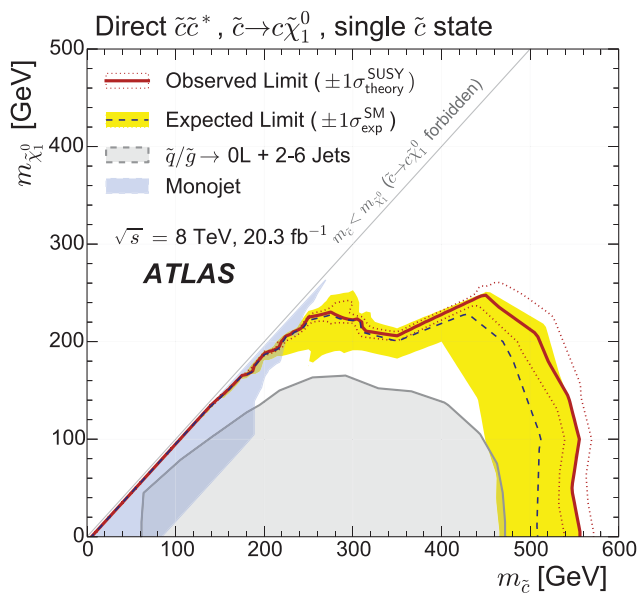

Figure 1. Exclusion limits at $95 \%$ confidence level (CL), placed in the $\left(\tilde{c}, \tilde{\chi_{1}^{0}}\right)$, mass plane (red), increasing the general exclusion placed by the ATLAS 0L+2-6 jets analysis (grey). Taken from Ref. [2].

\subsection{Stop via stau}

The decay processes of the SUSY particles are largely dependent upon the SUSY symmetry breaking scenario under consideration. In the case of gauge mediated symmetry breaking the LSP is the gravitino $(\tilde{G})$. Due to this, more complex decay modes are available such as the top squark decaying via an intermediate tau slepton $\tilde{\tau}$. Ref. [5] describes a search for stop pair production, where each stop decays via a stau $(\tilde{t} \rightarrow \tilde{\tau} b v)$, with the stau subsequently decaying to a tau and a gravitino $(\tilde{\tau} \rightarrow \tau \tilde{G})$. 
Three channels are investigated, differing by the decay mode for the tau pair that is under investigation, with each having dedicated SRs targeted at different regions of phase space. The main background is from $t \bar{t}$ pair production, with both real-tau, and mis-identified taus as separate components of this background. CRs are defined for both the real-tau and fake-tau $t \bar{t}$ contributions to produce separate normalisation parameters for $t \bar{t}$ pair production decaying into real taus and mis-identified taus.

No significant excesses are seen in any of the SRs, and a statistical combination of the SRs from each channel is performed to produce overall 95\% CL exclusion limits in the $(\tilde{t}, \tilde{\tau})$ mass plane, Fig. 2.

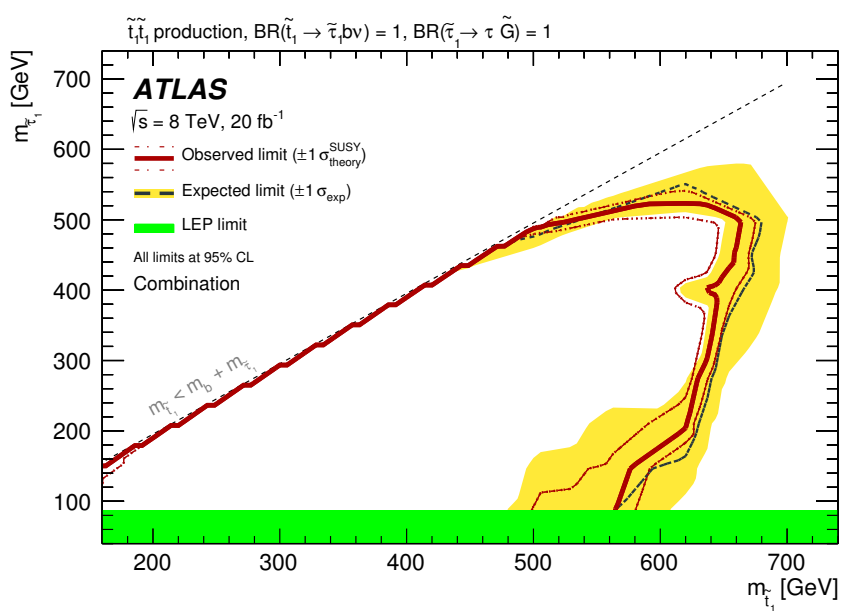

Figure 2. Exclusion limits at a 95\% CL from the stop to stau analysis in the $(\tilde{t}, \tilde{\tau})$ mass plane, assuming the process $\tilde{t} \rightarrow \tilde{\tau} b v$, and subsequently $\tilde{\tau} \rightarrow \tau \tilde{G}$. Taken from Ref. [5].

\subsection{RPV Stop}

R-parity violating SUSY scenarios do not contain the large $\mathrm{E}_{\mathrm{T}}^{\mathrm{miss}}$ which is characteristic of R-parity conserving models and hence must use different methods and variables in order to discriminate signal and background processes. A search for RPV stop pair production is presented in Ref. [6], searching for stop pair production with the top squark decaying via $\tilde{t} \rightarrow c s$. Earlier experiments place limits on stop masses below $100 \mathrm{GeV}$ in these scenarios [7].

The main discriminating variable in the analysis is the average jet mass of a large radius jet, where the radius $(\mathrm{R})$ is dependent upon the stop mass under consideration: The main background is multi-jet production, which is estimated using a purely data driven estimate. No excesses are found, and limits are placed on the stop mass in such scenarios, with $100<m_{\tilde{t}}<310 \mathrm{GeV}$ excluded, Fig. 3.

\section{Third Generation Summary Paper}

Throughout Run 1 of the LHC many searches have been performed for third generation squarks, with limits placed on a wide variety of different scenarios. In order to fully exploit these results, the Third Generation Summary paper [8] was produced, containing interpretations of SM analyses, investigations into additional decay modes and statistical combinations of existing analyses, to combine the results and futher constrain the SUSY parameter space. 


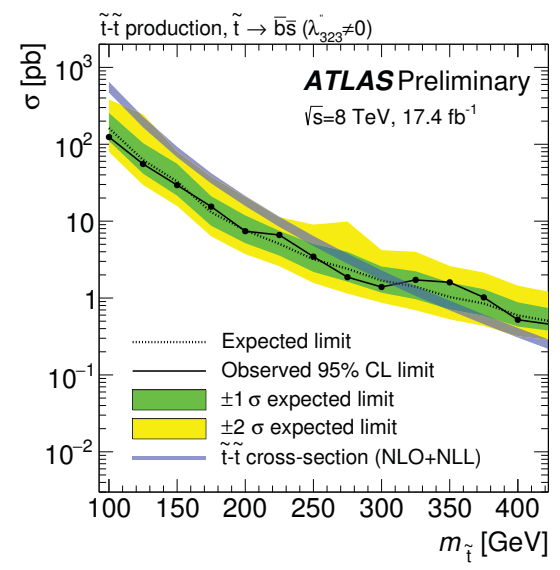

Figure 3. 95\% CL exclusion Limits placed on the stop mass by the RPV stop search. The search extends the limits set by earlier experiments and excludes $m_{\tilde{t}}$ up to $310 \mathrm{GeV}$. Taken from Ref. [6].

\subsection{Reinterpreting Existing Analyses}

Interpretation of SM measurements into the properties of the top quark can provide limits on the top squark mass, in regions of phase space that are difficult to target with conventional analysis techniques. The reinterpretation of both the ATLAS $t \bar{t}$ cross section measurement [9] and the spin correlation measurement (the opening angle $\Delta \phi(l, l)$ between the two leptons from dileptonic $t \bar{t}$ decays) [10] place limits in the region where $m_{\tilde{t}}=m_{t}$, due to the difference expected in the distributions if a top squark with $m_{\tilde{t}}=m_{t}$ is present. The combination of the exclusion from the cross section measurement and the spin correlation measurement (Fig. 4), allow limits to be placed on light top squarks, with 150 $<m_{\tilde{t}}<191 \mathrm{GeV}$ excluded, with the assumption of massless neutralinos.
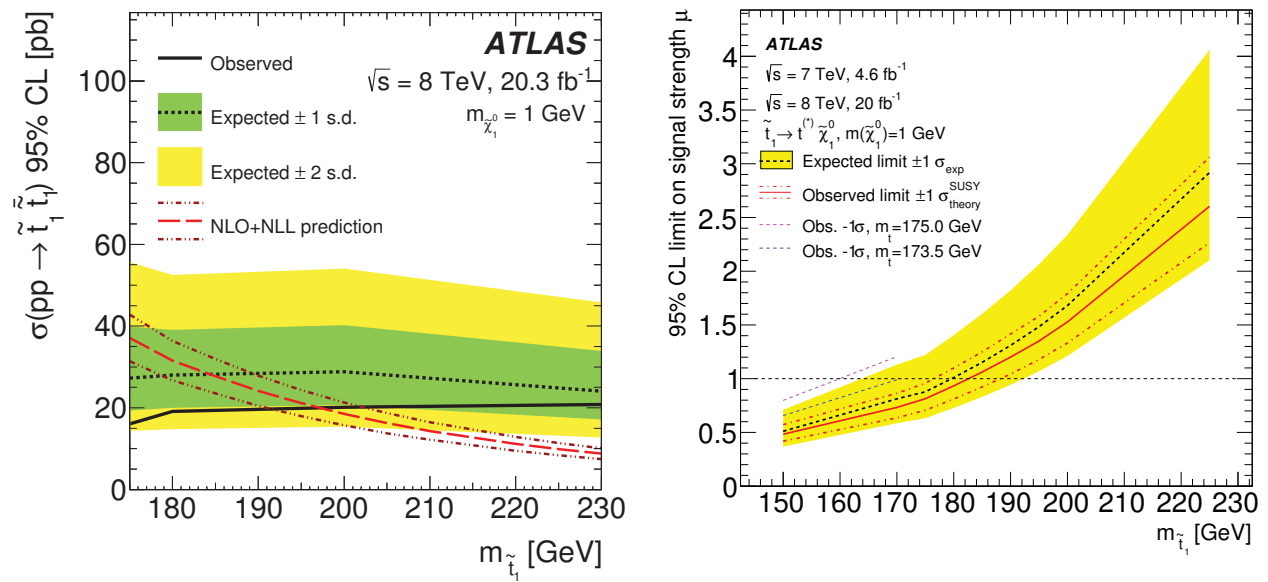

Figure 4. Left: Limits on $m_{\tilde{t}}$ from the reinterpretation of the SM $\sigma_{t \bar{t}}$ measurement. Right: Limits on $m_{\tilde{t}}$ from the reinterpretation of the $t \bar{t}$ spin correlation measurement. Taken from Ref. [8]. 


\subsection{Investigating Additional Decay Modes}

More complex decay scenarios are also considered, for example the "tbMET" and the "WW-like" analyses.

The tbMET analysis targets stop pair production where two possible decay channels are available for the stop, $\tilde{t} \rightarrow t+\tilde{\chi_{1}^{0}}$, and also decays involving charginos $\left(\tilde{\chi_{1}^{ \pm}}\right), \tilde{t} \rightarrow b+\tilde{\chi_{1}^{ \pm}}$(with the subsequent decay $\tilde{\chi_{1}^{0}} \rightarrow W^{*}+\tilde{\chi_{1}^{0}}$, as the neutralino and chargino are treated as almost mass degenerate in these scenarios). Various branching ratios of these decays are considered in the analysis, which is optimised for the $1 \ell+2 b$-jets $+\mathrm{E}_{\mathrm{T}}^{\text {Miss }}$ final state. The main SM backgrounds are $t \bar{t}$ and $W$-jets production. Branching ratio dependent limits are placed in the $\left(m_{\tilde{t}}, m_{\tilde{\chi_{1}^{0}}}\right)$ phase space, for the scenarios where $\Delta m\left(\tilde{\chi_{1}^{ \pm}}, \tilde{\chi_{1}^{0}}\right)$ $=5$ or $20 \mathrm{GeV}$, (Fig. 5).
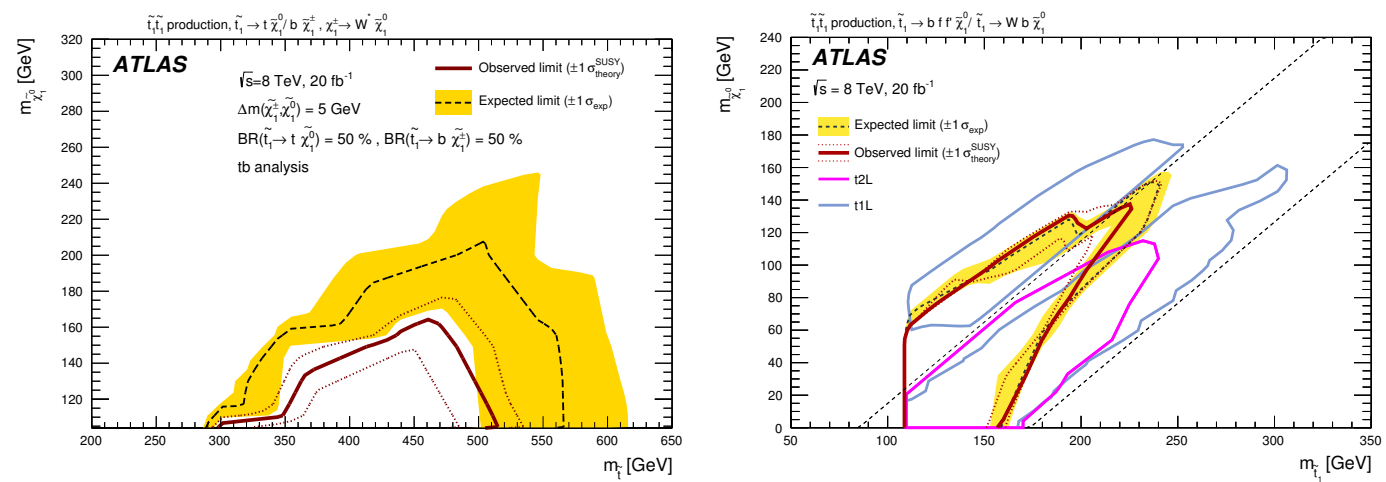

Figure 5. Left: Exclusion limits at $95 \% \mathrm{CL}$ placed in the $m\left(\tilde{t}, \tilde{\chi_{1}^{0}}\right)$ phase space by the tbMET analysis, for a $\operatorname{BR}\left(\tilde{t} \rightarrow b+\tilde{\chi_{1}^{ \pm}}\right)=50 \%$ and assuming $\Delta m\left(\tilde{\chi_{1}^{ \pm}}, \tilde{\chi_{1}^{0}}\right)=5 \mathrm{GeV}$. Right: Limits placed by the WW-like analysis which provides $95 \%$ exclusion limits in the region where the three and four body stop decay modes overlap. Taken from Ref. [8].

The WW-like analysis considers two possible mass spectra for the top squark, where either: the mass difference between the stop and neutralino being less than the $W$-boson mass $\left(m_{\tilde{t}}-m_{L S P}<m_{W}\right)$ which considers the four body stop decay, $\left.\tilde{t} \rightarrow b f f \tilde{\chi}_{1}^{0}\right)$; or a mass difference between the stop and neutralino being larger than the $W$ mass, but smaller than the top mass $\left(m_{W}<m_{\tilde{t}}-m_{L S P}<m_{t}\right)$ which considers a three body stop decay, $\tilde{t} \rightarrow b W{\tilde{\chi_{1}^{0}}}^{0}$. The analysis is motivated by the discrepancy between the SM expectation and the measurement of $\sigma_{W W}$ reported by both ATLAS [11] and CMS [12]. The analysis is optimised for a final state consisting of two different flavour (DF, $e \mu$ ) opposite sign (OS) leptons, with the stransverse mass $\left(\mathrm{m}_{\mathrm{T} 2}[13]\right)$ as the main discriminating variable. No significant excesses were found in any of the $7 \mathrm{SRs}$, and exclusion limits are placed in the $\left(m_{\tilde{t}}, m_{\tilde{\chi}_{1}^{0}}\right)$ mass plane, Fig. 5.

\subsection{Limits on the Heavier Stop Eigenstate}

An alternative method to investigate difficult to target regions of the stop phase space is to search for pair production of the heavier stop eigenstate $\left(\tilde{t}_{2}\right)$, which is a convienient method to search in the region where $\Delta m\left(\tilde{t}, \tilde{\chi_{1}^{0}}\right) \approx m_{t}$. Such decays are searched for in three channels: $\tilde{t_{2}} \rightarrow \tilde{t_{1}}+Z, \tilde{t_{2}} \rightarrow \tilde{t_{1}}+h$, 
$\tilde{t_{2}} \rightarrow \tilde{t_{1}}+\tilde{\chi_{1}^{0}}$, with many SRs defined for each. No excesses are seen in any of the channels and $95 \%$ CL exclusion limits are placed in the $m\left(\tilde{t_{2}}, \tilde{\chi_{1}^{0}}\right)$ phase space assuming $\mathrm{BR}=100 \%$, Fig. 6 .

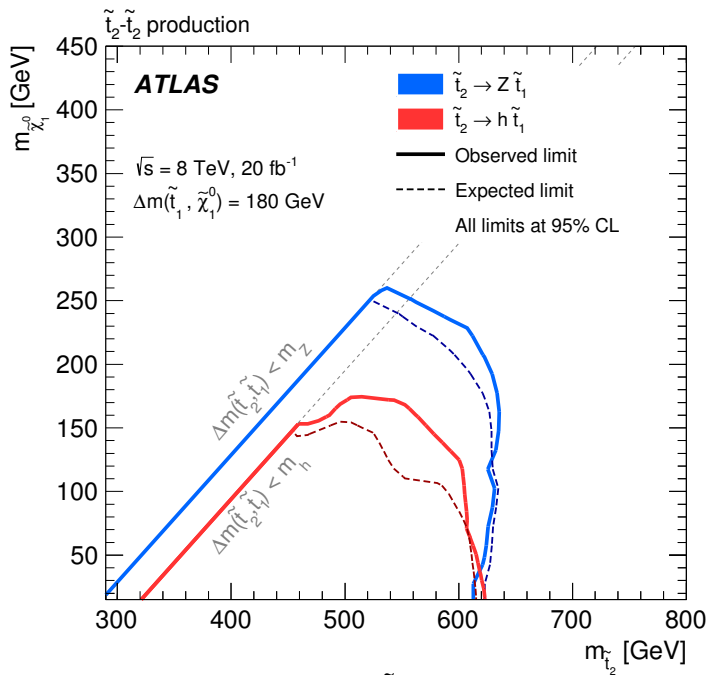

Figure 6. $95 \% \mathrm{CL}$ exclusion limits placed in the $m\left(\tilde{t_{2}}, \tilde{\chi_{1}^{0}}\right)$ phase space assuming BR $=100 \%$ for the decays considered. Taken from Ref. [8].

\subsection{Sbottom Limits}

Sbottom decay modes are also investigated, with dedicated analyses (such as the "bbMET" search for sbottom pair production with the sbottom decaying via: $\left.\tilde{b} \rightarrow \tilde{b \chi_{1}^{0}}[14]\right)$ and reinterpretations of other analyses (for example the reinterpretation the ATLAS same sign (SS) or $3 \ell$ strong production analysis [15] to investigate $\tilde{b} \rightarrow b \tilde{\chi}_{2}^{0}$, with $\left.\tilde{\chi_{2}^{0}} \rightarrow \tilde{\chi_{1}^{0}}+h\right)$. Limits are subsequently placed in the $m\left(\tilde{b}, \tilde{\chi_{1}^{0}}\right)$ phase space by these analyses, Fig. 7.

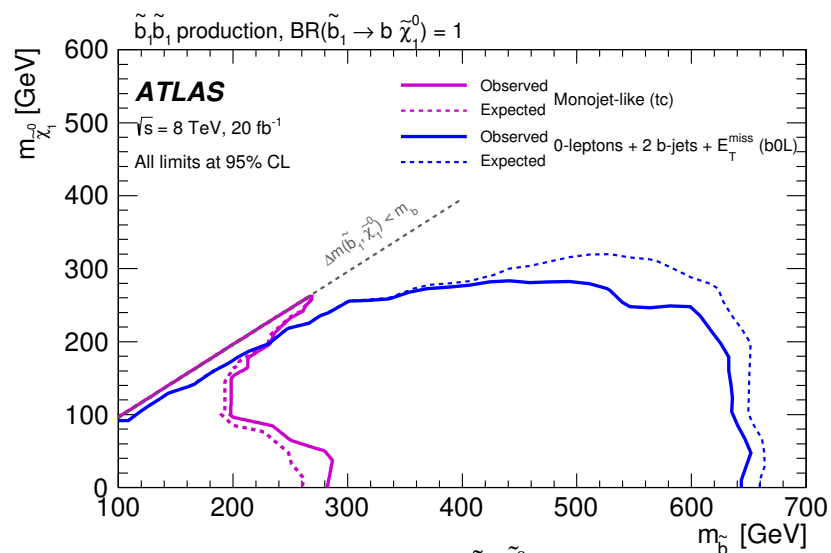

Figure 7. Exclusion limits at the $95 \% \mathrm{CL}$ placed in the $m\left(\tilde{b}, \tilde{\chi_{1}^{0}}\right)$ phase space by the bbMET analysis (blue) and the Monojet analysis (purple). Taken from Ref. [8]. 


\section{Outlook for LHC Run 2}

Many ATLAS third generation analyses are targeting discovery with Run 2 data, one of which is the sbottom analysis. The analysis has a similar strategy to the Run 1 sbottom analysis, focusing on final states with two $b$-tagged jets and missing transverse energy, to search for direct sbottom pair production with the sbottom decaying via $\tilde{b} \rightarrow b \tilde{\chi_{1}^{0}}$. Preliminary studies indicate that with an integrated luminosity of $10 \mathrm{fb}^{-1}, 3 \sigma$ evidence for bottom squarks with mass up to $800 \mathrm{GeV}$ may be obtained [16].

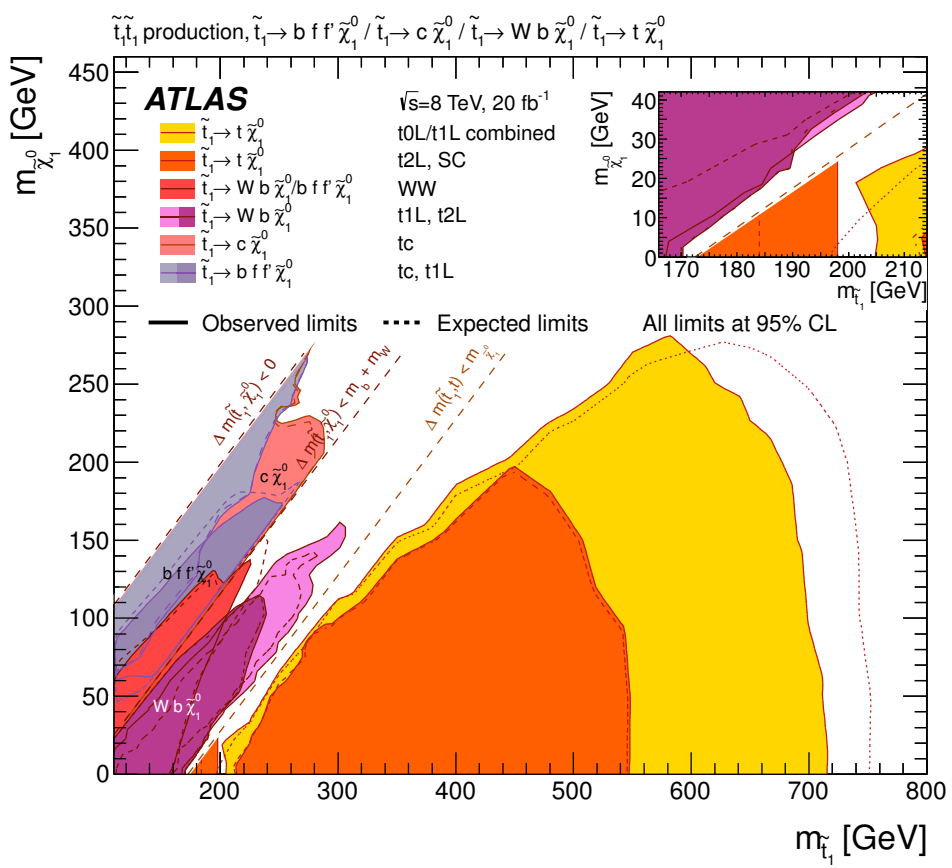

Figure 8. Exclusion limits at $95 \% \mathrm{CL}$ in the $\tilde{t}, \tilde{\chi_{1}^{0}}$ mass plane provided by the full programme of ATLAS third generation searches during LHC Run 1. Taken from Ref. [8].

\section{Conclusion}

ATLAS is almost complete with its dedicated program of searches for third generation squarks during Run 1 of the LHC, with limits placed on a wide variety of SUSY scenarios. Fig. 8 shows the full extent of the coverage and exclusion in the $\left(\tilde{t}, \tilde{\chi_{1}^{0}}\right)$ mass plane that has been provided by Run 1 ATLAS searches, with similar coverage and exclusion also in the $\left(\tilde{b}, \tilde{\chi_{1}^{0}}\right)$ mass plane. data.

Run 2 preparation is well underway, targeting discovery of third generation squarks with early 


\section{References}

[1] R. Barbieri and G. Giudice, Nucl. Phys. B 306 (1988) 63-76.

[2] The ATLAS Collaboration, Phys. Rev. Lett. 144, 161801 (2015) arXiv:1501.01325 [hep-ex].

[3] D. R. Tovey, JHEP 0804 (2008) 034, arXiv:0802.2879 [hep-ph].

[4] The ATLAS Collaboration, JHEP09 (2014) 176 arXiv:1405.7875 [hep-ex].

[5] The ATLAS Collaboration, submitted to EPJC, arXiv:1509.04976 [hep-ex].

[6] The ATLAS Collaboration, ATLAS-CONF-2015-026.

[7] CDF Collaboration, T. Aaltonen et al, Phys. Rev. Lett. 111 (2013) 031802, arXiv:1303.2699 [hepex].

[8] The ATLAS Collaboration, submitted to Eur. Phys. J, arXiv:1506.08616 [hep-ex].

[9] ATLAS Collaboration, JHEP 1406 (2014) 035, arXiv:1404.2500 [hep-ex].

[10] ATLAS Collaboration, Eur. Phys. J. C74 no. 10, (2014) 3109, arXiv:1406.5375 [hep-ex].

[11] ATLAS Collaboration, Phys. Lett. B712 (2012) 289-308, arXiv:1203.6232 [hep-ex].

[12] CMS Collaboration, Eur. Phys. J. C73 no. 10, (2013) 2610, arXiv:1306.1126 [hep-ex].

[13] A. Barr, C. Lester, and P. Stephens, J.Phys. G29 (2003) 2343-2363, arXiv:hep-ph/0304226 [hep-ph].

[14] The ATLAS Collaboration, JHEP 10 (2013) 189, arXiv:1308.2631 [hep-ex].

[15] The ATLAS Collaboration, JHEP 06 (2014) 035, arXiv:1404.2500 [hep-ex].

[16] The ATLAS Collaboration, ATL-PHYS-PUB-2015-005. 